\title{
Automatic Labeling of Anatomical Structures in MR FastView Images Using a Statistical Atlas
}

\author{
Matthias Fenchel ${ }^{1,2}$, Stefan Thesen ${ }^{1}$, and Andreas Schilling ${ }^{2}$ \\ ${ }^{1}$ Siemens Healthcare Sector, MR Division, Applications \& Workflow, \\ Erlangen, Germany \\ matthias.fenchel.ext@siemens.com \\ ${ }^{2}$ WSI-GRIS, Eberhard-Karls-Universität, Tübingen, Germany
}

\begin{abstract}
We present a method for fast and automatic labeling of anatomical structures in MR FastView localizer images, which can be useful for automatic MR examination planning. FastView is a modern MR protocol, that provides larger planning fields of view than previously available with isotropic 3D resolution by scanning during continuous movement of the patient table. Hence, full 3D information is obtained within short acquisition time. Anatomical labeling is done by registering the images to a statistical atlas created from training image data beforehand. The statistical atlas consists of a statistical model of deformation and a statistical model of grey value appearance. It is generated by non-rigid registration and principal component analysis of the resulting deformation fields and registered images. Labeling of an unseen FastView image is done by non-rigid registration of the image to the statistical atlas and propagating the labels from the atlas to the image. In our implementation, the statistical models of deformation and appearance are both implemented on the GPU (graphics processing unit), which permits computing the atlas based labeling using GPU hardware acceleration. The running times of about 10 to 30 seconds are of the same magnitude as the image acquisition itself, which allows for practical usage in clinical MR routine.
\end{abstract}

\section{Introduction}

In current clinical MR practice, it is desirable to have more automation and standardization of the examination workflows. Automation of manual tasks like slice positioning, coil selection, adaptations of sequence parameters, etc. would reduce the need for highly trained technical operators. It would also lead to more reproducibility, more comparability and shorter examination slots, which in turn increases the overall utilization of the scanner. Solutions based on image processing from fast pre-scan acquisitions for the automation of manual steps are highly appreciated. To name but a few of these methods, van der Kouwe et al. 1] presented a solution for reproducible MR head scan planning using a human brain atlas. Peschl [2] showed a method for the spine. In [3] a method for automatic liver scan planning from fast localizer scans was published.

D. Metaxas et al. (Eds.): MICCAI 2008, Part I, LNCS 5241, pp. 576-584, 2008

(C) Springer-Verlag Berlin Heidelberg 2008 
This paper presents an algorithm for fast and automatic labeling of anatomical structures in fast MR whole-body pre-scans. We aim at a complete labeling or multi-organ segmentation of the patient's torso for an automation of the MR exam with respect to complex positioning, marking, reading and reporting features. The performance of the applications should be competitive with a human operator in terms of speed and precision. This requires a fast imaging protocol and a fast segmentation algorithm. The FastView protocol is a good basis for the anatomical labeling with the purpose of the desired applications as it provides full 3D information, large field of view, sufficient resolution (5 mm isotropic) and fast acquisition (about 20 seconds). It is a modern proton-density weighted $2 \mathrm{D}$ axial acquisition technique during continuous movement of the patient table similar to [4] and [5]. Keil et al. 6] already used this protocol for an estimation of the patient's position and orientation.

The labeling resp. segmentation algorithm uses a statistical atlas of deformation and appearance of the human torso, which captures the averages and main modes of variation in shape and intensity. The atlas is created from a set of representative training data instances. Registration of an unseen data set to the statistical atlas, which is accomplished by finding the deformation field and appearance that 'best' fits the data set, yields the anatomical labeling or multi-organ segmentation by label propagation.

Atlas based segmentation has been abundantly used throughout medical image segmentation literature. Most publications have been made in human brain image segmentation and diagnosis. Talairach presented an atlas with a complete reference coordinate system for the human brain [7. Cootes introduced active shape models and active appearance models and published their application to atlas based matching in [8]. Rueckert proposed statistical analysis of the using non-rigid deformation fields for the creation of a statistical atlas 9] of the brain. Zhou and Bai [10] created an abdominal atlas from CT scans and used it in a fuzzy-connectedness approach for identification of abdominal organs. Park et al. 11] used a probabilistic abdominal atlas for supervised multi-organ segmentation from CT scans. To the best of our knowledge this paper is the first to create an atlas from whole-body MR images of the complete human torso, although with moderate resolution, and use it for multi-organ segmentation or anatomical labeling.

\section{Building the Statistical Atlas}

\subsection{Training Data Acquisition and Preprocessing}

For the statistical atlas, a representative group of 31 volunteers is scanned using the FastView protocol. The images are acquired with a resolution of $5 \mathrm{~mm}$ isotropic and an axial field of view of $1000 \mathrm{~mm}$. The training group is selected in a way that ensures that a possibly large portion of natural variation is captured, which means that volunteers of different ages, sex and stature are scanned 
using different scanner devices with different hardware and $B_{0}$ field strengths. The images are first cropped and transformed to a common coordinate frame. As the arm positions of the volunteers during the acquisition are arbitrary, which can have harmful effects on the non-rigid registrations, the images are preprocessed with an automatic arm stripping algorithm. Further processing is done with pure torso images, as shown in figure 2 . The image intensities are also normalized by piecewise linear scaling such that the peaks of the two-modal histograms coincide.

\subsection{Non-rigid Inter-subject Registration}

Modeling natural variation requires establishing spatial correspondence among the training image data sets from the volunteers. This is done implicitly by performing non-rigid registrations of all data sets to a reference data set.

The non-rigid transformation is set up by a global affine transformation and a local free-form transformation. For the free-form transformation, a regular 3D control grid is superimposed onto the image. The deformation of a single point is given by the deformations of the control grid with a cubic B-spline tensor product interpolation. This ensures both smoothness and local support of the deformation coefficients, which is similar to the deformation used by Rueckert et al. in 9]. The similarity measure of choice for inter-subject registration of MR images is mutual information [12], based upon minimizing histogram entropy and allowing for variations of contrast among different subjects.

The registration process is driven by a a cost function, which combines a normalized mutual information term $N M I$ with an additional regularization term summing up the squared norm of differences of adjacent control point shifts $v_{i}$, which is similar to the well-known diffusion regularizer. This regularization term punishes 'unnatural' or rupturing deformations. A third term considers predefined correspondences, so called manual landmarks $L M$. The cost function reads as follows with the empirical constants $\lambda$ and $\gamma$ :

$$
\operatorname{Cost}(T)=N M I+\lambda \sum_{(i, j) \in N B}\left\|v_{i}-v_{j}\right\|^{2}+\gamma \sum_{x_{i} \in L M}\left\|x_{i}^{\prime}-T\left(x_{i}\right)\right\|^{2}
$$

where $T$ is the non-rigid deformation, $N B$ is the 27-neighborhood on the grid of control points, $v_{i}$ is the movement of control point $i, L M$ is the set of all manual landmarks, $x_{i}^{\prime}$ is a landmark in the reference image and $x_{i}$ in the data image. $\lambda$ and $\gamma$ are empirical coefficients that weight the different terms.

The cost function is minimized using a gradient descent optimization with adaptive step length. This procedure increases the step length as long as the value decreases steadily, but decreases its step length if not. The registration is implemented using a multi-scale approach, starting with a coarse resolution of image data and control grid points and gradually refining the image data and the grid resolution. The maximum resolution of the control grid is $20 \mathrm{~mm}$, i.e. one grid point for 4 voxels. 

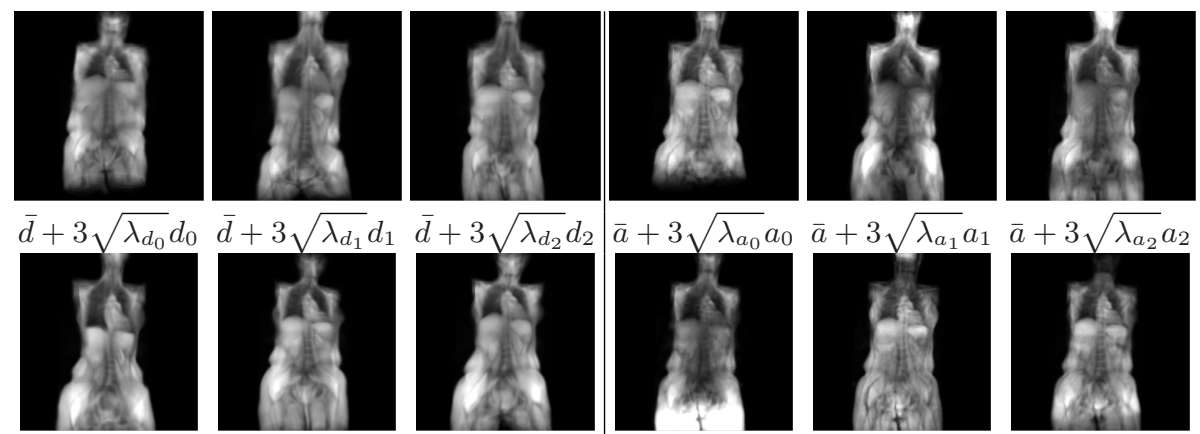

$\bar{a}+3 \sqrt{\lambda_{a_{2}}} a_{2}$

$\bar{d}-3 \sqrt{\lambda_{d_{0}}} d_{0} \bar{d}-3 \sqrt{\lambda_{d_{1}}} d_{1}$
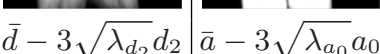

$\bar{a}-3 \sqrt{\lambda_{a_{1}}} a_{1}$

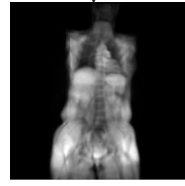

Fig. 1. This figure shows the major principal modes of deformation (first 3 columns) and appearance (second 3 columns) of the atlas. $\bar{d}$ is the average deformation, $\bar{a}$ the average intensity and $\lambda_{i}$ the respective principal values $i$, with $\sqrt{\lambda_{i}}$ being the Gaussian standard deviation of mode $i$.

\subsection{Statistical Model of Deformation}

Registering all image data sets $I_{i}$ to a reference data set $I_{r e f}$ produces $n$ non-rigid transformations $T_{i}$ and $n$ registered data sets $I_{i}\left(T_{i}\right)$. Each of the transformations $T_{i}$ contains an affine part $M_{i}$ and a free-form part $d_{i}$, with the coefficients of the control grid point shifts. Since statistical analysis is to be performed exclusively on the free-form part, all affine content is removed from the $d_{i}$ beforehand.

Statistical models of deformation [9] extend the concepts of active shape models. Instead of performing statistical analysis on point coordinates, principal component analysis is directly calculated on the deformation fields $d_{i}$. This yields the linear model:

$$
d_{\text {instance }}=\bar{d}+\Phi_{d} \cdot d
$$

where $\bar{d}$ is the average deformation field, $\Phi_{d}$ a matrix whose columns contain the principal deformations and $d$ the feature vector with the weights of the principal components. Setting the entries of the feature vector $d$ to a range within $\pm 3 \sqrt{\lambda_{i}}$ produces valid deformation fields within a $\pm 3 \sigma_{i}$ interval of the implicit multivariate Gaussian distribution. Those deformation fields can be used to warp unseen image data for registration to the atlas. Figure 1 shows images of the final atlas and the statistical model of deformation.

\subsection{Statistical Model of Appearance}

The variety of the intensity appearances of identical organs in different subjects is handled by a statistical model of appearance, which is set up by performing principal component analysis on the pixelwise intensity values of the registered data sets $I_{i}\left(T_{i}\right)$. In contrast to active appearance models [13] or [14, however, the appearance statistics are kept independent of the deformation model without correlation. This is motivated by the fact that the intensity appearance of an 
image may be strongly influenced by external effects like the actual scanner hardware, e.g. coils, the basic magnetic field etc. and not only by the proton densities of the actual organs. As many of these effects are obviously independent of the deformation fields, they should be treated differently in order to keep the atlas free from biases. The last three columns of figure 1 show three modes of the statistical model of appearance.

\section{Application of the Atlas for Fast Anatomical Labeling}

Labeling resp. segmentation of an unseen data set is done by registering the data set to the statistical atlas and propagating the anatomical labels from the atlas to the unseen data set. First, an outline of the registration algorithm is given followed by a description of a fast implementation using programmable GPU hardware.

\subsection{Atlas Based Registration}

The labeling algorithm consists of 3 phases: First, a rough affine offset registration of the unseen image $I_{u n s}$ to the statistical atlas is computed by aligning the bounding boxes of binary images created from the data. Next, the affine transformation $M$, the instance of the deformation field $d$ and the intensity appearance $a$ of the atlas are calculated which best map $I_{\text {uns }}$ to the atlas $I_{\text {atlas }}$. Finally, the labels of the anatomical structures are propagated to $I_{\text {uns }}$ using the registration result.

The registration result is found by minimizing the following distance cost function:

$$
\left(M_{\text {opt }}, d_{\text {opt }}, a_{\text {opt }}\right)=\arg \min _{M, d, a} \frac{\sum_{x_{i} \in \Omega} w_{i}\left(I_{\text {atlas }}\left(a, x_{i}\right)-I_{\text {uns }}\left(T\left(x_{i}\right)\right)\right)^{2}}{\sum_{x_{i} \in \Omega} w_{i}}
$$

where $x_{i}$ are the world coordinates of pixel $i$ and $w_{i}$ a specific weight for the influence of pixel $i$ to the registration. This is particularly important for certain structures of high variation, like head, legs or stomach, which should not affect organs with small variation like the lungs. If $w_{i}$ is set to $\frac{1}{\sigma\left(x_{i}\right)^{2}}$, the squared standard deviation of each pixel in the training images, a Gaussian distance between the atlas and $I_{\text {uns }}$ is obtained. Final division by the total sum of weights normalizes the distance measure with respect to the overlap size of $I_{\text {atlas }}$ and $I_{\text {uns }}$.

The iterative registration algorithm repeats the following steps: First, an intensity instance $I_{\text {atlas }}$ of the atlas is created using the appearance feature values $a_{i}$. An instance $d$ of the deformation field is computed from the deformation feature values $d_{i}$. The unseen image $I_{\text {uns }}$ is warped using the affine offset $M$ and the free-form deformation $d$ followed by the computation of the distance measure between $I_{a t l a s}$ and $I_{\text {uns }}$. This is repeated until the optimum has been found. 

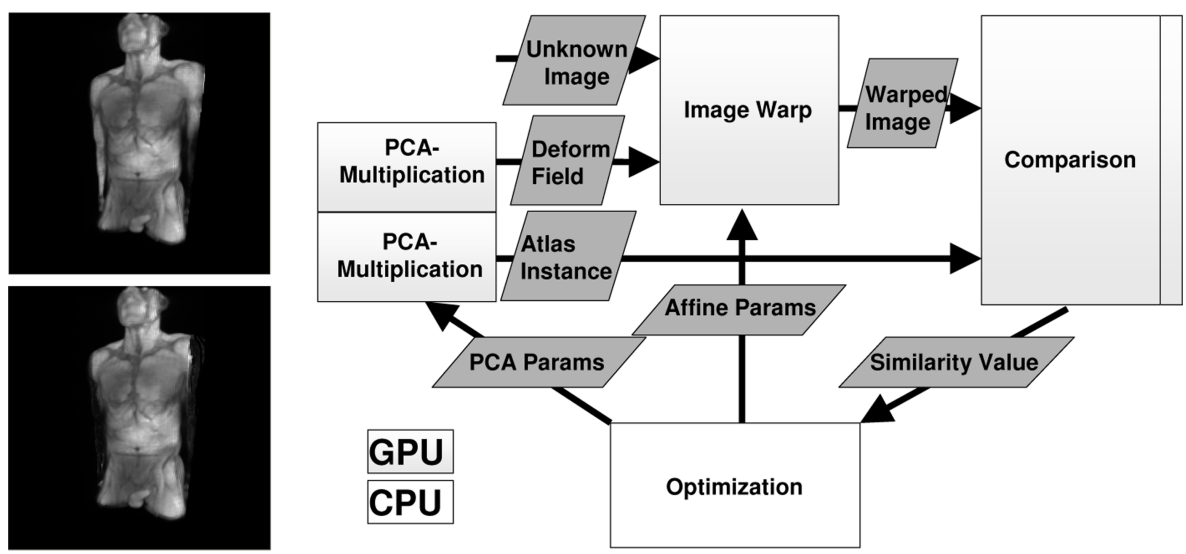

Fig. 2. Top shows Fig. 3. Data flow and computation components of the registraFastView image in tion algorithm. The white box indicates the CPU based compuvolume rendering, tation component, while the boxes shaded in grey indicate GPU bottom the result based computation components.

after arm stripping

The registration runs in a hierarchical top-down way, starting with a coarse resolution and gradual refinement. A Gauss-Newton optimization scheme is chosen, with numerical derivatives being computed using symmetric differences. Gauss-Newton requires the image to be divided into blocks for which the distance measure values are calculated in parallel.

\subsection{GPU Based Implementation}

A basic implementation of the registration algorithm is slow, because each warping step requires B-spline tensor computations for each voxel. However, modern computer graphics hardware provides enormous floating-point, parallel computation and interpolation power off the shelf. This potential has proven to be powerful for general purpose computations like image registration [15]. Although warping the images by means of a fragment shader on the GPU results in an enormous performance boost, each warp requires the deformation field being transferred from memory to the graphics card and the warped volume being transferred back, which unnecessarily slows down the computation. Therefore, all image based computation steps are implemented as fragment shaders on the GPU, including the generation of a deformation field instance, (i.e. evaluating equation 2), generation of an intensity instance of the atlas, warping and evaluation of the distance function. This reduces data transfer between GPU and memory to the parameter values of the optimization and the values of the similarity measure resp. cost function. See figure 3 for an overview of the data flow and components of computation. All data needed for the registration, i.e. atlas data, image data, matrix data, etc. are stored using float textures in the GPU memory, which minimizes data traffic and boosts performance. 


\section{Results and Discussion}

Validation of the atlas based segmentations is done by placing anatomical landmarks in all data sets. Between 2 and 4 landmark points per organ of interest are used. Reproducibility is studied by repeating the placements multiple times and with different operators. The mean positions of these landmarks are then considered to be the ground truth. The standard deviations were about 10.4 $\mathrm{mm}$. The performance of the atlas based registrations and labelings is assessed by calculating the displacements of the propagated landmark positions to the ground truth. All tests are performed in leave-all-in and leave-one-out scenarios. Leave-all-in means that the atlas is built from all data sets and then compared to the result of the registration of one of those data sets. Table 1 gives an overview of the results.
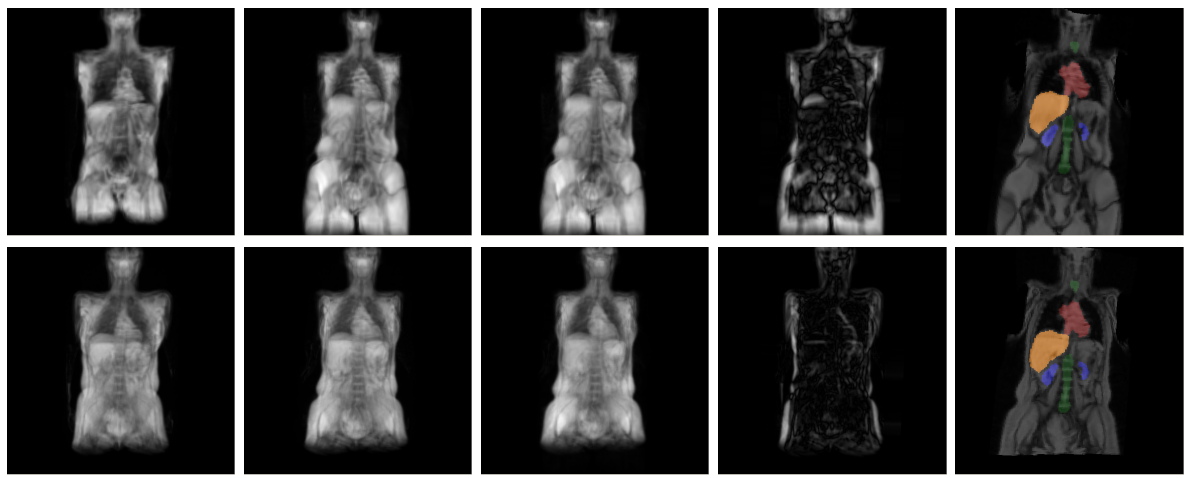

Fig. 4. This figure shows two registration results: from left to right, the original images, the warped images, the best fitting instances of the atlas, difference images and slice images of propagated labels for heart, kidneys, spine and liver

Table 1. The experimental results of the displacements between the manual landmarks and the landmarks determined by the atlas based registration

\begin{tabular}{|c|c||c|c|}
\hline \# Deformation modes & \# Appearance modes & \multicolumn{2}{|c|}{$\begin{array}{c}\text { LM displacements (mm) } \\
\text { leave-all-in }\end{array}$} \\
\hline \hline 0 & 5 & 23.7 & 28.5 \\
10 & 5 & 17.4 & 25.4 \\
20 & 10 & 13.7 & 24.0 \\
30 & 15 & 11.7 & 22.4 \\
\hline
\end{tabular}

Average calculation times are about 10-30 seconds depending on the number of modes being used. Evidently, the results improve with an increasing number of modes. Overall, the deviations converge to a $\sigma$ resp. $2 \sigma$ interval of the ground truth for the leave-all-in resp. leave-one-out case. 


\section{Conclusion and Future Work}

We presented a method for fast anatomical labeling in FastView MR localizer images. The results are promising and suitable for the desired applications. As for further applications, more exactness may be needed, future work will focus on improving the data basis of the atlas, which will lead to an atlas with more generalization ability. Considering its leave-one-out performance, the atlas would certainly benefit from additional training data. Another issue will be to further enhance performance by switching from numerical to analytical derivatives calculated on the GPU. Future work will also consider a final fine-tuning segmentation of the anatomical labels, using local histogram measures and shape priors. Currently, the search space of the registration is constrained to the space of valid deformation instances of the atlas. In the future this could be extended to a free non-rigid registration guided by a deformation prior imposed by the statistical model of deformation.

\section{References}

1. van der Kouwe, A., Benner, T., Fischl, B., Schmitt, F., Salat, D., Harder, M., Sorensen, A., Dale, A.: On-line automatic slice positioning for brain MR imaging. NeuroImage 27(1), 222-230 (2005)

2. Peschl, S., Ernst, T., Speck, O., Hennig, J.: Auto alignment of intervertebral disk. In: Proceedings of the 12th Annual Meeting of the ISMRM, vol. 2216 (May 2004)

3. Fenchel, M., Thesen, S., Schilling, A.: Fully automatic liver scan planning - slice and navigator positioning from stacked $2 \mathrm{D}$ localizer scans. In: Proceedings of the 14th Annual Meeting of ISMRM 2006, p. 467 (2006)

4. Barkhausen, J., Quick, H., Lauenstein, T., Goyen, M., Ruehm, S., Laub, G., Debatin, J., Ladd, M.: Whole-body MR imaging in 30 seconds with real-time TRUEFISP and a continuously rolling table platform: feasibility study. Radiology 220, 252-256 (2001)

5. Kinner, S., Zielonka, A., Zenge, M.O., Ladd, S., Ladd, M.: Whole-body MR imaging with continuously moving table and multiplanar reformations: toward parameter optimization for SSFP imaging in patient examinations. In: Proceedings of the 14th Annual Meeting of ISMRM 2006, vol. 2421 (2006)

6. Keil, A., Wachinger, C., Brinker, G., Thesen, S., Navab, N.: Patient position detection for SAR optimization in magnetic resonance imaging. In: Larsen, R., Nielsen, M., Sporring, J. (eds.) MICCAI 2006. LNCS, vol. 4191, pp. 49-57. Springer, Heidelberg (2006)

7. Talairach, J., Tournoux, P.: Co-planar Stereotaxic Atlas of the Human Brain: 3Dimensional Proportional System - an Approach to Cerebral Imaging. Thieme Medical Publishers, New York (1988)

8. Cootes, T.F., Beeston, C., Edwards, G.J., Taylor, C.J.: A unified framework for atlas matching using active appearance models. In: Kuba, A., Sámal, M., ToddPokropek, A. (eds.) IPMI 1999. LNCS, vol. 1613, pp. 322-330. Springer, Heidelberg (1999)

9. Rueckert, D., Frangi, A.F., Schnabel, J.A.: Automatic construction of 3D statistical deformation models of the brain using non-rigid registration. IEEE Trans. Med. Imaging 22(8), 1014-1025 (2003) 
10. Zhou, Y., Bai, J.: Atlas based automatic identification of abdominal organs. Proceedings of the SPIE Medical Imaging 5747, 1804-1812 (2005)

11. Park, H., Bland, P.H., Meyer, C.R.: Construction of an abdominal probabilistic atlas and its application in segmentation. IEEE Trans. Med. Imaging 22(4), 483$492(2003)$

12. Wells, W., Viola, P., Atsumi, H., Nakajima, S., Kikinis, R.: Multi-modal volume registration by maximization of mutual information. In: Medical Image Analysis, pp. 35-51 (1996)

13. Cootes, T.F., Edwards, G.J., Taylor, C.J.: Active appearance models. In: European Conference on Computer Vision, vol. 2, pp. 484-498 (1998)

14. Stegmann, M.B., Ersbøll, B.K., Larsen, R.: Fame - a flexible appearance modelling environment. IEEE Trans. Med. Imaging 22(10), 1319-1331 (2003)

15. Harris, M.: GPGPU: General purpose computation on GPUs. In: Eurographics (2004) 